\title{
POLICONSUMO SIMULTÁNEO DE DROGAS EN ESTUDIANTES DE PREGRADO DEL ÁREA DE LA SALUD DE UNA UNIVERSIDAD, SAN SALVADOR - EL SALVADOR
}

\author{
Fabio Bautista Pérez', Laura Simich'2, Carol Strike ${ }^{3}$,Bruna Bradns ${ }^{4}$, Norman Giesbrecht ${ }^{5}$, Akwatu Khenti ${ }^{6}$
}

\footnotetext{
${ }^{1}$ MSc. Investigador. Dirección de Investigación. Vice-Rectoría de Investigación. Universidad Evangélica de El Salvador. San Salvador, El Salvador. E-mail: fabio.bautista@uees.edu.sv.

2 Ph.D. Senior Scientist. Research Scientist. Health Systems Research and Consulting Unit. Centre for Addiction and Mental Health. University of Toronto. Toronto, Canada. E-mail: laura_simich@camh.net.

${ }^{3}$ Ph.D. Researcher Scientist. Health System Research and Consulting Unit. Center for Addiction and Mental Health. University of Toronto. Toronto, Canada. E-mail: carol_strike@camh.net

${ }^{4}$ Ph.D. Senior Scientist. Office of Research and Surveillance. Drug Strategy and Controlled Substances Programme. Health Canada and Public Health and Regulatory Policies. Center for Addiction and Mental Health. University of Toronto, Toronto, Canada. E-mail: bruna_brands@camh.net

${ }^{5}$ Ph.D. Researcher. Health Systems Research and Consulting Unit. Centre for Addiction and Mental Health. University of Toronto, Canada. E-mail: norman_giebsbrecht@camh.net

${ }^{6}$ MSc. Director of Office of International Health Center for Addiction and Mental Health. Toronto, Canada, E-mail: akwatu_ khenti@camh.net
}

RESUMEN: El objetivo del estudio fue describir los patrones de policonsumo simultáneo de drogas en estudiantes de pregrado de una universidad de la ciudad de San Salvador, El Salvador. Estudio de diseño transversal y análisis descriptivo, con un tamaño de la muestra de 309 estudiantes. Un 5.18\% de los estudiantes reporto policonsumo simultaneo en los últimos 12 meses, cifra que se reduce a $4.21 \%$ para los últimos 30 días. La edad promedio de inicio del policonsumo fue 16.9 años. En los últimos 12 meses y 30 días, la combinación más usada entre los estudiantes fue alcohol + tabaco con $2.3 \%$. Las características de consumo más referidas fueron: el aprendizaje individual/por sí mismo, el consumo en grupos mixtos, fuera de la universidad/en clubes nocturnos y el hacerlo para la relajación.

DESCRIPTORES: Estudiantes. Drogas ilictas. Conducta adictiva. Trastornos relacionados con sustancias. Factores de riesgo.

\section{SIMULTANEOUS POLYDRUG USE AMONG UNDERGRADUATE STUDENTS OF HEALTH SCIENCIES FROM ONE UNIVERSITY, SAN SALVADOR - EL SALVADOR}

\begin{abstract}
The objective of the study was to describe patterns of simultaneous polydrug use among undergraduate students at one university in the city of San Salvador, El Salvador. The study was cross-sectional, with a descriptive analysis, with a sample size of 309. Simultaneous polydrug use was reported by $5.18 \%$ of the students for the last 12 months, and $4.21 \%$ for the last 30 days. The average onset age for polydrug use was 16.9 years. In the last 12 months and 30 days, the most common combination by used by the students was alcohol + tobacco $2.3 \%$. The characteristics of consumption most referred were: learning individually by themselves, consumption in gender mixed groups, out side university in nightclubs, and to do it for relaxing.
\end{abstract}

DESCRIPTORS: Students. Street drugs. Addictive behavior. Substance-related disorders. Risk factors.

\section{POLICONSUMO SIMULTÂNEO DE DROGAS EM ALUNOS DE GRADUAÇÃO DA ÁREA DE CIÊNCIAS DA SAÚDE EM UMA UNIVERSIDADE, SAN SALVADOR - EL SALVADOR}

RESUMO: O objetivo foi descrever padrões de policonsumo de drogas em estudantes de graduação em uma universidade em San Salvador, El Salvador. A pesquisa foi descritiva, com um desenho tranversal, com o tamanho da amostra foi de 309 estudantes. Verificouse que 5,18\% dos alunos tinham usado mais de uma substância psicoativa, simultaneamente, nos últimos 12 meses, enquanto nos últimos 30 dias o valor foi reduzido para 4,21\%. A idade média de início de consumo de poli foi de 16,9 anos. Nos últimos 12 meses e 30 dias, a combinação mais comumente usada entre os estudantes foi álcool + tabaco 2,3\%. A forma mais utilizada de aprendizagem era individual, consumidos em grupos mistos, fora da faculdade/clubes, e para relaxar.

DESCRITORES: Estudantes. Drogas ilícitas. Comportamento aditivo. Transtornos relacionados ao uso de substancias. Fatores de risco. 


\section{INTRODUCCIÓN}

A nivel regional existe una creciente preocupación por los estudiantes universitarios de América Latina y el Caribe, debido a que cada vez más, el consumo de drogas pone en peligro su bienestar, al participar en comportamientos de riesgo, lo que aumenta la carga social del cuidado en cada nación, por el gasto que se requiere para los pacientes, costo económico, servicios de salud y pérdida de la productividad como consecuencia del abuso de sustancias psicoativas. ${ }^{1-3}$

La tendencia creciente del uso indebido de sustancias entre los estudiantes universitarios se considera una importante crisis de salud pública para algunas naciones. De acuerdo con el estudio "Desperdiciar lo mejor y lo más brillante: el abuso de sustancias en colegios y universidades de América", las principales drogas de abuso en la universidad y los campus universitarios es el alcohol $65 \%$ - 70\%. Aunque la principal droga consumida en los campus sigue siendo el alcohol $49.4 \%$, el uso de otras drogas lícitas e ilícitas se ha incrementado. En la actualidad los estudiantes que usan marihuana y otras sustancias ilegales se han duplicado, las cifras pasan de $1.9 \%$ a $4.0 \%$ por ciento, mientras que el uso de otras drogas como la cocaína aumentó en un 52\%. La tendencia señalada se ha intensificado en los últimos decenios y ahora los estudiantes están inmersos en una cultura de abuso de sustancias adictivas que se traduce en una serie de efectos nocivos en el ámbito académico, la salud y en consecuencias sociales que se extienden a las comunidades circundantes. ${ }^{4-11}$

Existen muy pocos datos disponibles sobre el uso indebido de drogas en estudiantes universitarios y estos se centran en el consumo de alcohol y los problemas relacionados en la universidad y estudiantes universitarios. Además, la mayoría de las investigaciones disponibles se concentran en la utilización de una única sustancia como el alcohol o la marihuana; con muy poca atención prestada al uso simultáneo de varias drogas. Esto es especialmente cierto para los países en desarrollo y regiones como el Caribe y América Latina. En El Salvador es el primer estudio que se realiza sobre policonsumo simultáneo de drogas en estudiantes universitarios, por lo que la información obtenida sobre este patrón de consumo será de mucha utilidad, ayudando a incrementar la información sobre el consumo de drogas en los estudiantes universitarios.

Para los efectos de este estudio el término "patrones" se referirá a la combinación de sus- tancias utilizadas, la frecuencia de consumo de drogas, marco temporal del uso de drogas y la diferencia entre sexos en la forma en que las drogas se utilizan. La pregunta de investigación formulada fue: ¿Cuáles son los patrones de poli consumo simultáneo de drogas y las implicaciones de género, legales y sociales en los estudiantes universitarios de pregrado en salud en una universidad de San Salvador, El Salvador?

El objetivo planteado fue comprender los patrones de policonsumo simultáneo y sus implicaciones de género, legales y sociales en estudiantes universitarios de pregrado en una universidad de San Salvador, El Salvador.

\section{MÉTODO}

Este estudio fue parte de una investigación multicéntrica en estudiantes de pregrado de carreras del área de la salud en seis países de América Latina y uno del Caribe. El estudio se realizó en una universidad de San Salvador. El diseño fue de tipo transversal y el análisis descriptivo. La universidad seleccionada tenía una población de 3700 estudiantes, con 1800 pertenecientes a la Facultad de Medicina, la que imparten tres carreras: medicina, nutrición y dietética, y enfermería. La población del estudio estuvo conformada por estudiantes de pregrado de primero y segundo año, matriculados a tiempo parcial o completo.

La muestra quedo constituida por 309 estudiantes, distribuidos por carrera de la siguiente manera: Medicina, 239 estudiantes, Nutrición y Dietética, 44 estudiantes y Enfermería, 26 estudiantes.

Para la recolección de la información se utilizó un cuestionario y la técnica de encuesta. Algunas preguntas del cuestionario fueron tomadas de escalas ya existentes como Reasons for Drugs $^{12}$ CAGE Questionary ${ }^{13}$ y Canadian Survey ${ }^{14}$. El cuestionario final quedó conformado por cuatro secciones y 58 preguntas cerradas que comprendía características socio-demográficas y sobre el uso de sustancias psicoactivas.

Para que todos los estudiantes tuvieran igual oportunidad de ser incluidos en el estudio, los cursos incluidos fueron seleccionados aleatoriamente. Los estudiantes elegibles fueron informados de los objetivos del estudio y del proceso para garantizar la confidencialidad. La participación fue voluntaria y se requirió la firma de consentimiento informado. Los datos fueron recolectados en las aulas de clase, y la administración de la encuesta 
fue llevada a cabo por el investigador principal. Una vez que el participante llenó los formularios los depositó en dos cajas separadas.

Los datos fueron analizados descriptivamente, incluidos distribución de frecuencias. ${ }^{15}$ Para el análisis de datos se utilizó el programa estadístico SPSS, Versión 15.0 para Windows. ${ }^{16-17}$

El estudio contó con la autorización de las autoridades la Universidad, y fue aprobado por la Oficina de Ética en Investigación del Centro de Adicciones y Salud Mental de Canadá (Protocol reference \#225/2008) y por el Comité de Ética de la Investigación de la Universidad Evangélica de El Salvador (Acta n. 3, 7 nov 2008).

\section{RESULTADOS}

\section{Características sociodemográficas de la muestra}

La muestra estuvo constituida por 309 estudiantes, siendo el 80,9\% de sexo femenino,

con un promedio de edad de 19.6 años. La distribución por carrera fue: Medicina, $77.3 \%$; $\mathrm{Nu}-$ trición y Dietética, $14.2 \%$; y Enfermería, $8.4 \%$. Un $90.5 \%$ estudiaban a tiempo completo, la mayoría no trabajaban $94.2 \%$, eran solteros $97.7 \%$, y vivían con sus familias $79.6 \%$. Un $20.4 \%$ no vivía con familiares. Respecto a la valoración de la religión, un $74.4 \%$ la consideró muy importante. El porcentaje de padres con educación de nivel universitario fue de $55.7 \%$ para los padres y $50.5 \%$ para las madres.

\section{Características generales del consumo de sustancias psicoactivas}

Un 25,2\% de los estudiantes mencionó haber consumido sustancias psicoactivas al menos una vez en la vida, un 18,8\% en los últimos 12 meses, y un $22.0 \%$ en los últimos 30 días. En todas estas categorías de tiempo predominó el consumo de alcohol y tabaco. La edad promedio de inicio en el consumo fue de 15.1 años.

La tabla 1 muestra la prevalencia en el uso por sustancia. Los datos muestran que el alcohol y el tabaco fueron las drogas que presentaron los mayores índices de consumo, especialmente alcohol, con cifras de $14.9 \%, 10.7 \%$, y $10.0 \%$ de consumo en los últimos 12 meses, 30 días y el último fin de semana respectivamente. Sólo un $2.9 \%$ reportó el consumo semanal, y nadie consumo diario. El tabaco fue la segunda sustancia más usada, con un 10.4 y $8.4 \%$ de los estudiantes reportando consumo en los últimos 12 meses y 30 días respectivamente. El uso semanal alcanzó un $4.2 \%$ y el consumo diario un $2.3 \%$.

El reporte del consumo de drogas ilícitas como la marihuana, cocaína, éxtasis y heroína fue bajo; lo mismo para inhalantes y drogas de prescripción. Se observo algún grado de inconsistencia en el reporte de uso de estas drogas.

Tabla 1 - Prevalencia en el uso de sustancias psicoactivas lícitas e ilícitas entre estudiantes de pregrado del área de salud, en una universidad de San Salvador-El Salvador, 2009 (n=309)

\begin{tabular}{|c|c|c|c|c|c|c|c|c|c|c|}
\hline \multirow{3}{*}{ Sustancias psicoactivas } & \multicolumn{10}{|c|}{ Prevalencia } \\
\hline & \multicolumn{2}{|c|}{$\begin{array}{c}\text { Últimos } 12 \\
\text { meses }\end{array}$} & \multicolumn{2}{|c|}{$\begin{array}{c}\text { Últimos } 30 \\
\text { días }\end{array}$} & \multicolumn{2}{|c|}{ Semanal } & \multicolumn{2}{|c|}{ Diaria } & \multicolumn{2}{|c|}{ Fin de semana } \\
\hline & $\mathbf{F}$ & $\%$ & $\mathbf{F}$ & $\%$ & $F$ & $\%$ & $\mathbf{F}$ & $\%$ & $\mathbf{F}$ & $\%$ \\
\hline Tabaco & 32 & 10.4 & 26 & 8.4 & 13 & 4.2 & 7 & 2.3 & 14 & 4.5 \\
\hline Alcohol & 46 & 14.9 & 33 & 10.7 & 9 & 2.9 & - & - & 31 & 10.0 \\
\hline Marihuana & 4 & 1.3 & 6 & 1.9 & 3 & 1.0 & - & - & 1 & 0.3 \\
\hline Cocaína & - & - & - & - & - & - & - & - & 1 & 0.3 \\
\hline Éxtasis & - & - & 1 & 0.3 & 1 & 0.3 & 1 & 0.3 & 1 & 0.6 \\
\hline Heroína & - & - & - & - & 1 & 0.3 & 1 & 0.3 & 1 & 0.6 \\
\hline Inhalantes & 2 & 0.6 & 1 & 0.3 & 1 & 0.3 & - & - & - & - \\
\hline Drogas prescritas & 5 & 1.6 & 3 & 1.0 & 1 & 0.3 & 1 & 0.3 & 1 & 0.3 \\
\hline
\end{tabular}

\section{Características del policonsumo simultáneo de sustancias psicoactivas}

El 5.18\% de los estudiantes mencionó haber utilizado más de una sustancia psicoactiva al mis- mo tiempo en los últimos 12 meses, mientras que en los últimos 30 días, esta cifra se redujo a $4.21 \%$. La edad promedio del inicio de policonsumo fue de 16.9 años. 
La tabla 2 resume las estadísticas de policonsumo. El consumo simultáneo alcohol + tabaco presentó los porcentaje más altos, con $2.3 \%$ de reporte en 12 meses y 30 días. Para las combinaciones de alcohol + marihuana, alcohol + drogas de prescripción, alcohol + tabaco + marihuana, alcohol + cocaína + tabaco, marihuana + cocaína y marihuana + drogas de prescripción, los porcentajes de consumo reportado oscilaron de 0.3 y 0.6\%. Para las combinaciones de alcohol + cocaína, alcohol + cocaína + marihuana + tabaco, tabaco + marihuana y tabaco + drogas de prescripción no se reportó ningún consumo.

Tabla 2 - Policonsumo simultáneo de sustancias lícitas e ilícitas entre estudiantes de pre-grado del área de la salud en una universidad de San Salvador-El Salvador, 2009 (n=309)

\begin{tabular}{lcccc}
\hline \multirow{2}{*}{ Policonsumo de sustancias psicoactivas } & \multicolumn{2}{c}{ Últimos $\mathbf{1 2}$} & meses & \multicolumn{2}{c}{ Últimos $\mathbf{3 0}$ días } \\
\cline { 2 - 5 } & $\mathbf{F}$ & $\mathbf{\%}$ & $\mathbf{F}$ & $\mathbf{\%}$ \\
\hline Alcohol + marihuana & 1 & 0.3 & 1 & 0.3 \\
Alcohol + cocaína & - & - & - & - \\
Alcohol + drogas prescritas & 2 & 0.6 & 1 & 0.3 \\
Alcohol + tabaco + marihuana & 1 & 0.3 & 2 & 0.6 \\
Alcohol + cocaína + tabaco & 2 & 0.6 & - & - \\
Alcohol + cocaína + marihuana + tabaco & - & - & - & - \\
Tabaco + marihuana & - & - & - & - \\
Tabaco + drogas prescritas & - & - & - & - \\
Marihuana + cocaína & 1 & 0.3 & 1 & 0.3 \\
Marihuana + drogas prescritas & 2 & 0.6 & 1 & 0.3 \\
Alcohol + tabaco & 7 & 2.3 & 7 & 2.3 \\
\hline
\end{tabular}

En relación a la forma en que los estudiantes aprendieron acerca de la combinación de sustancias psicoactivas, la forma más referida fue el aprendizaje por mí mismo $4.2 \%$, seguido por la alternativa "por medio de un amigo" $2.6 \%$. Los lugares donde se realiza este tipo de consumo fueron, en orden decreciente: fuera de la universidad/ en clubes nocturnos $4.2 \%$, fuera de la universidad $2.6 \%$ y otros sitios $1.6 \%$, como la casa, en fiestas de casas de los amigos, en calles y parques, la playa y montañas. Un $8.1 \%$ manifestó adquirir las sustancias fuera del campus.

Un $6.1 \%$ refirió consumir en grupos mixtos; un $2.9 \%$ refirió conseguir dichas sustancias por ellos mismos; con su propio dinero $5.5 \%$ o solicitado a un miembro de la familia $2.3 \%$. La decisión del tipo de sustancia y la combinación de éstas para consumirlas fue decidida en forma individual $3.6 \%$.

Los estudiantes perciben como fácil 30.7\% o muy fácil $23.0 \%$ el acceso a las sustancias psicoactivas ilícitas, pero el $43.0 \%$ manifestó no tener conocimiento de ello.

En relación al acceso de los estudiantes a las drogas de prescripción cuando no tienen una prescripción médica, el $45.6 \%$ mencionó que fue a través de los amigos y $12.6 \%$ a través de un proveedor. El 35.9\% dijo no saber.

En cuanto a la percepción que el estudiante tiene sobre el consumo de sustancias psicoactivas en la universidad, el $27.5 \%$ mencionó que es un problema muy importante para la Universidad, el $25.5 \%$ piensa que no es un problema y el $38.5 \%$ no lo sabe.

El consumo de sustancias psicoactivas por los profesionales del área de la salud fue considerado por los estudiantes como un gran problema por el $64.1 \%$, y como un problema menor por el $12.0 \%$ de los encuestados. El 16.5\% dijo no saber.

La tabla 3 muestra la frecuencia de respuesta referente a las razones que induce el consumo de sustancias psicoactivas en forma simultánea. Los aspectos centrales más referidos fueron propósitos sociales y cambio de ánimo. Las razones especificas referidas con más frecuencias fueron: "le ayuda a relajarse" 5.8\% y "le ayuda a disfrutar la compañía de sus amigos(as)" 5.5\%.

Es importante mencionar que un porcentaje muy bajo de estudiantes $0.6 \%$ manifestó haber sido presionado para iniciar el consumo simultaneo de sustancias psicoactivas, y el 1,3\% refirió haber influido en otra persona para iniciar el policonsumo. 
Tabla 3 - Razones seleccionadas por los estudiantes de pregrado de la área de la salud para usar sustancias psicoactivas. San Salvador-El Salvador, 2009 (n=309)

\begin{tabular}{|c|c|c|c|}
\hline Aspecto central & Razones & $\mathbf{F}$ & $\%$ \\
\hline \multirow{5}{*}{ Cambio de ánimo } & Le hace sentirse mejor cuando está deprimido & 8 & 2.6 \\
\hline & Le ayuda a dejar de preocuparse por un problema & 10 & 3.2 \\
\hline & Le ayuda a relajarse & 18 & 5.8 \\
\hline & Le ayuda a sentir emoción o euforia (para experimentar) & 8 & 2.6 \\
\hline & Para sentirse fuertemente drogado o intoxicado & 2 & 0.6 \\
\hline \multirow{4}{*}{ Efectos físicos } & Mejora las sensaciones durante las relaciones sexuales & 7 & 2.3 \\
\hline & Le ayuda a permanecer despierto & 12 & 3.9 \\
\hline & Le ayuda a perder peso & 3 & 1.0 \\
\hline & Le ayuda a dormir & 9 & 2.9 \\
\hline \multirow{7}{*}{ Propósitos sociales } & & 17 & 5.5 \\
\hline & Le ayuda a disfrutar la compañía de sus amigos(as) & & \\
\hline & Le ayuda a sentir más confianza o ser más capaz de hablar con las personas en & 9 & 2.9 \\
\hline & una situación social & 6 & 1.9 \\
\hline & Le ayuda a desinhibirse & 10 & 3.2 \\
\hline & Le ayuda a mantenerse bien cuando sale en la noche con sus amigos(as) & & \\
\hline & Para relacionarse mejor con los demás debido a la presión social & 6 & 1.9 \\
\hline \multirow{2}{*}{$\begin{array}{l}\text { Facilitación de } \\
\text { actividades }\end{array}$} & Le ayuda a concentrarse en el trabajo o en el estudio & 4 & 1.3 \\
\hline & $\begin{array}{l}\text { Incrementa las actividades como escuchar música, jugar o practicar un deporte } \\
\text { Le ayuda a hacer algo cuando está aburrido }\end{array}$ & 5 & 1.6 \\
\hline \multirow{2}{*}{$\begin{array}{l}\text { Manejo de los } \\
\text { efectos de otras } \\
\text { sustancias }\end{array}$} & & ? & 10 \\
\hline & Le ayuda a atenuar los efectos posteriores de otras sustancias & 3 & 1.0 \\
\hline
\end{tabular}

\section{DISCUSIÓN}

La tendencia creciente en el consumo de sustancias psicoactivas entre estudiantes universitarios ha sido considerado como una crisis en la salud pública. Esto genera especial preocupación debido a las potenciales consecuencias para el futuro desarrollo del país. ${ }^{1,4}$

De acuerdo a las características sociodemográficas el promedio de edad de los estudiantes era de 19,6 años, la mayoría era del género femenino, solteros, viven en casa con sus familiares, son estudiantes a tiempo completo y consideran a la religión muy importante.

El consumo de sustancias psicoactivas una vez en vida, en los últimos 12 meses y en los últimos 30 días fue considerado como moderadamente alto. El inicio del consumo de sustancias psicoactivas fue en el rango de 14 a 18 años, coincidente con otros estudios nacionales e internacionales. ${ }^{5,18}$ Un 25.2\% mencionó haber consumido drogas alguna vez en la vida, un 18,8\% en los últimos 12 meses y un 22,0\% en los últimos 30 días.

Las sustancias psicoactivas lícitas más consumidas en los últimos 12 meses y últimos 30 días fueron el alcohol y el tabaco, pero en cantidades más bajas a las reportadas internacionalmente. ${ }^{4-8}$
En cuanto a sustancias ilícitas, como la marihuana, cocaína, éxtasis y heroína; y otras como inhalantes y drogas de prescripción, el consumo fue mucho más bajo a lo reportado en Estados Unidos, México, Sudamérica, Europa y Australia. Estudios nacionales por otro lado muestra cifras más cercanas a las nuestras. ${ }^{4-11,18}$

Algo similar fue observado con las cifras de reporte de policonsumo simultaneo, de 5,2\% en los últimos 12 meses y de 4,2\% en los últimos 30 días, cifras menores a las reportadas en otras investigaciones internacionales..$^{9,19-21}$

La edad promedio del inicio para el consumo general fue de 15,1 años y de 16,9 años para el policonsumo simultáneo. Esto está en línea con lo planteado en la literatura, donde se describe que el consumo de sustancias psicoactivas es iniciado desde muy temprana edad, en la escuela secundaria, antes de iniciar sus estudios universitarios. ${ }^{5,9,18-21}$

El mayor policonsumo simultáneo de drogas correspondió al alcohol + tabaco, lo que concuerda con estudios realizados en otros países. ${ }^{1,9,19,22}$ En los casos de alcohol + marihuana, alcohol + drogas de prescripción, alcohol + tabaco + marihuana, alcohol + cocaína + tabaco, marihuana + cocaína y marihuana + drogas de prescripción, los 
porcentajes de consumo reportados fueron bajos, y no sobrepasaron el $0,6 \%$. Esto no coincide con reportes internacionales, donde las cifras de consumo presentadas son más elevadas. ${ }^{1,9,21}$

Sería importante realizar este tipo de investigaciones en otros centros educativos del país, incluyendo los niveles secundarios, de modo de conocer la situación de la problemática relacionada al consumo de drogas desde una perspectiva más amplia. Esto podría ayudar a plantear estrategias de prevención y combate más efectivas.

Las fuentes principales de estrés o ansiedad para los estudiantes fueron las tareas y horarios universitarios, problemas económicos, familiares, de trabajo y las relaciones de pareja. A pesar de ello, consideran que tienen un buen desempeño académico, con promedios de notas buenos y excelentes. También manifiestan tener muy buenas relaciones con sus padres. Los estudiantes dicen aprender por sí mismos o por medio de un amigo, las sustancias

son consumidas fuera de la universidad, conseguidas por ellos mismos, con su propio dinero y fuera de la universidad. La mayoría considera que el acceso a dichas sustancias es fácil. Los grupos donde los estudiantes consumen sustancias psicoactivas son predominantemente mixtos.

En los aspectos centrales "propósitos sociales" y "cambio de ánimo" se concentran razones más citadas para el consumo, tales como "le ayuda a relajarse" y "le ayuda a disfrutar la compañía de sus amigos(as)". Otros investigadores mencionan que los principales motivos para el consumo de drogas fueron la sensación de placer. ${ }^{19}$

El modelo del estudio planteado en base a la teoría bio-psico-social ha sido coherente con los factores involucrados con el policonsumo, donde la religión/creencias, fuentes de estrés o ansiedad y relación con sus padres puede influenciar el patrón de consumo de sustancias psicoactivas.

Consideramos de mucha preocupación que los futuros profesionales de la salud puedan tener alguna adicción al consumo de drogas, por las consecuencias que ello puede tenerse en el desempeño de su profesión, así como en su propia vida y el cuidado de la salud de la población. En consecuencia, nos parece prioritario promover estrategias que coadyuven al mejoramiento de los métodos de prevención existente y a establecer nuevas alianzas que permitan reducir al mínimo esta problemática.

\section{CONCLUSIONES}

El estudio permitió observar características de policonsumo de sustancias psicoactivas, lícitas e ilícitas, en estudiantes de pregrado del área de salud de las carreras de Medicina, Nutrición y Dietética, y Enfermería, en una universidad de El Salvador. Se observó que la prevalencia de consumo no simultáneo fue moderadamente alta, siendo el alcohol y tabaco los más consumidos. El reporte de consumo de drogas ilícitas fue bajo. En relación al policonsumo simultáneo, se observaron cifras bajas, siendo la combinación alcohol + tabaco la que resultó con el mayor porcentaje. Consideramos necesaria la implementación de programas de prevención del consumo y policonsumo simultáneo de drogas lícitas e ilícitas, para obtener una población de estudiantes libres de consumo de drogas.

\section{Limitaciones}

Los resultados obtenidos en este estudio no son generalizables a la población de jóvenes en general o a la de estudiantes universitarios, ya que sólo se incluyeron estudiantes de primero y segundo años de las carreras de Medicina, Nutrición y Dietética, y Enfermería en una universidad en El Salvador.

\section{Recomendaciones}

La Dirección de Proyección Social de la Universidad deberá implementar programas de inducción sobre la prevención del consumo y policonsumo simultáneo de drogas lícitas e ilícitas, para obtener una población de estudiantes libres de consumo de drogas. Promover estrategias que motiven a los estudiantes a participar en actividades grupales como deportes, arte y cultura, con el fin de alejarlos de las drogas. Al CICAD deberá continuar promoviendo nuevas investigaciones en el policonsumo simultáneo de sustancias psicoactivas a nivel de las universidades latinoamericanas, donde se incluyan todas las carreras que sirven las universidades. La Comisión Nacional Antidrogas del país deberá sugerir desarrollo de políticas a las universidades sobre la problemática de las drogas y apoyar a los programas de prevención sobre el consumo de drogas que las universidades estén implantando.

\section{AGRADECIMIENTOS}

Al gobierno de Canadá/DFAIT, Organización de los Estados Americanos (OEA), Comisión Interamericana para el Control del Abuso de Drogas (CICAD), Centre for Addiction and Mental Health (CAMH - Toronto/Canadá). Asimismo, se agradece a los estudiantes de pregrado que participaron en el estudio y a todas las personas que colaboraron de forma directa o indirecta en la 
implementación del estudio. Un agradecimiento especial al Dr. Gustavo Mery, CAMH Reviewer, por su colaboración en la revisión y editoración final de todos los trabajos y informes del grupo III.

\section{REFERENCIAS}

1. Engs R, Hansen DJ. Boozing and brawling on campus: a national study of violent problems associated with drinking over the past decade. J Crim Justice. 1994; 22(2):171-80.

2. Klingemann S. A risky combination? adolescents' drinking patterns and academic performance. Conferencia presentada a la reunion annual de la American Sociological Association [online], Atlanta Hilton Hotel, Atlanta, EE.UU. Jamaica, 2008 [cited 2008 Ago 24]. Disponible en: http://www.enotes. com/world-fact-book/jamaica-jm

3. Perkins HW. Surveying the damage: a review of research on consequences of alcohol misuse in college populations. J Stud Alcohol Suppl [online]. 2002 Mar [cited 2008 Jun 20]; (14):91-100. Disponible en: http:/ / www.collegedrinkingprevention.gov/ media/Journal/091_139.pdf

4. National Centre on Addiction and substance abuse, Columbia University. Wasting the best and the brightest: Substance abuse at America's colleges and universities. Casa. Columbia, EE.UU. 2007.

5. Comisión Nacional Antidrogas. Estudio de prevalencia y patrones de consumo de sustancias lícitas e ilícitas en salvadoreños entre las edades de 12 a 71 años. CNA. San Salvador: Comisión Nacional Antidrogas; 2005.

6. Gómez A, Herde,J, Laffee A, Lobo S, Martín E. Consumo de drogas lícitas e ilícitas por estudiantes universitarios. Rev Facu Ciencias Salud Universidad de Carabobo [online]. 2007 [acceso 2008 Jul 17]; 11(3):41-5. Disponible en: http:/ / bases.bireme.br/ cgibin/wxislind.exe/iah/online/?IsisScript=iah/ iah. $x$ is\&src $=$ google\&base $=$ ADOLEC\&lang $=p \& n e x$ tAction $=\operatorname{lnk} \&$ exprSearch $=502852$ \&indexSearch $=$ ID

7. Carlini E, Galduroz J, Noto A, Nappo A. II Levantamento domiciliary nacional sobre o uso de drogas psicotrópicas: estudo envolvendo as 108 maiores cidades do país [online]. National Institute of drugs abuse [cited 2008 Jul]. Available from: http:/ / www. unodc.org/pdf/brazil/ / II\% 20Levantamento \% 20 Domiciliar\%20Dr\%20Elisaldo\%Carlini_alterado2.pdf

8. Maddux J, Hoppe S, Costello RM. Psichoactive substance use among medical student. American J Psychiatry [online] 2006 [cited 2008 Jul 15], 143(2):187-91. Disponible en: http:/ / www.ncbi.nlm. nih.gov/pubmed/3946652

9. McCabe SE, Cranford JA, Morales M, Young A. Simultaneous and concurrent poly-drug use of alcohol and prescription drugs: prevalence, correlates, and consequences. J Stud Alcohol. 2006 Jul; 67(4):529-37.

10. Organización de los Estados Americanos y Comisión Interamericana para el Control del Abuso de Drogas. El Salvador: evaluación del progreso de control de drogas, 2005-2006. mecanismo de evaluación multilateral (MEM). Washington (US): OEA; 2006.

11. The Survey System. Sample Size Calculator. Creative Research System [online]. [cited 2008 Sep 10] 2008. Disponible en: http:/ / www.surveysystem.com/ sscalc.htm

12. Boys A, Marsden, J, Strang, J. Understanding reason for drug use among young people: a functional perspective. Health Educ Res. 2001 Aug; 16(4):457-69.

13. Erwing J. Detecting alcoholism: the CAGE questionnaire. JAMA. 1984 Oct 12; 252(14):1905-7.

14. Centre of Addiction and Mental Health and Université de Montreal. Canadian Campus Survey 2004. Toronto (CA): CAMH, GRSP,CIHR-IRC; 2004.

15. Hernández R, Fernández C, Baptista P. Metodología de la investigación en ciencias sociales. $4^{\mathrm{a}}$ ed. Mexico D.F (MX): McGraw-Hill; 2008.

16. Ferrán Aranaz M. SPSS para Windows. Análisis estadísticos. Madrid (ES): Mc Graw Hill; 2004.

17. Pérez López C. Técnicas estadísticas con SPSS 12: aplicaciones y análisis de datos. Madrid (ES): Pearson Prentice; 2005.

18. Rivas de Rio R, Jenner C. Consumo de sustancias psicoactivas lícitas (alcohol y tabaco), en estudiantes masculinos y femeninos de 16 a 45 años de edad entre el primero y quinto año de estudio durante el ciclo 01 del año 2004 en la Universidad Francisco Gavidia de San Salvador [tesis online]. El Salvador (SV): Universidad Francisco Gavidia, Facultad de Jurisprudencia y Ciencias Sociales, Escuela de Psicología. 2004. Disponible: http/tesis/ / www.gobernación.gob.sv/observatorio/demanda2004/estudios/tesis\%UFG.pdf

19. Webb E, Ashton CH, Kelly P, Kamali F. Alcohol and drug use in UK university student. Lancet. 1996 Oct 5; 348(9032):922-5.

20. Ashton E. Alcohol abuse makes prescription drug abuse more likely [online]. National Institute of drugs abuse. 2008 [cited 2008 Jul 15]. Disponible en: www.drugabuse. gov/NIDA_notes/NNvol21N5/alcohol.html

21. O'Reily BO, Jessen J. Cannabis and other drug use by tertiary students in Darwin, Northern Territory, Australia. South Pacific J Psychol [online]. 2005; 16(1): [acceso 2008 Out 1]. Disponible en: http:// spjp.massey.ac.nz/issues/2005-v16/v16-oreilly.pdf

22. Monshouwer K, Smit F, Verdurmen J. Cannabis in the context of polidrug use: results from the Dutch National Shool Survey [online]. 2008 [cited 2008 Jul 15] Disponible en: http/ / www.emcdda.europa.eu/ publications/monographs/cannabis 